\title{
No attenuation of gastric distress or benefit to performance with adaptation to octanoate-rich esterified oils in female cyclists
}

Thorburn, Megan S.; Vistisen, Bodil; Thorp, Rhys M.; Rockell, Mike J.; Jeukendrup, Asker E.; Xu, Xuebing; Rowlands, David S.

\author{
Published in: \\ European Journal of Sport Science
}

Link to article, DOI:

$10.1080 / 17461390701673684$

Publication date:

2007

Document Version

Early version, also known as pre-print

Link back to DTU Orbit

Citation (APA):

Thorburn, M. S., Vistisen, B., Thorp, R. M., Rockell, M. J., Jeukendrup, A. E., Xu, X., \& Rowlands, D. S. (2007). No attenuation of gastric distress or benefit to performance with adaptation to octanoate-rich esterified oils in female cyclists. European Journal of Sport Science, 7(4), 179-192. https://doi.org/10.1080/17461390701673684

\section{General rights}

Copyright and moral rights for the publications made accessible in the public portal are retained by the authors and/or other copyright owners and it is a condition of accessing publications that users recognise and abide by the legal requirements associated with these rights.

- Users may download and print one copy of any publication from the public portal for the purpose of private study or research.

- You may not further distribute the material or use it for any profit-making activity or commercial gain

- You may freely distribute the URL identifying the publication in the public portal 
No attenuation of gastric distress or benefit to performance with adaptation to octanoate-rich esterified oils in female cyclists

Megan S. Thorburn, ${ }^{1}$ Bodil Vistisen, ${ }^{3,4}$ Rhys M. Thorp, ${ }^{1}$ Mike J. Rockell, ${ }^{2}$ Asker E. Jeukendrup, ${ }^{5}$ Xuebing $\mathrm{Xu}^{6}$, and David S. Rowlands ${ }^{1}$

Institute of Food, Nutrition, and Human Health, Massey University, Wellington, ${ }^{1}$ and Palmerston North ${ }^{2}$, New Zealand

The Copenhagen Muscle Research Centre, ${ }^{3}$ Department of Human Physiology, Institute of Exercise and Sport Sciences, 4 Department of Medical Physiology, Panum Institute, University of Copenhagen, Copenhagen, Denmark

${ }^{5}$ School of Sport and Exercise Sciences, University of Birmingham, Birmingham, United Kingdom

${ }^{6}$ BioCentrum-DTU, The Technical University of Denmark, Lyngby, Denmark

Correspondence:

Dr David Rowlands

Institute of Food, Nutrition, and Human Health

Massey University

PO Box 756, Wellington

New Zealand

$\mathrm{Ph}+6448012794$ ext 6940

FAX +64 48014994 
d.s.rowlands@massey.ac.nz

Running title: MCFA adaptation and performance in females 


\begin{abstract}
We investigated the effects of modifying a normal dietary fatty-acid composition and the ingestion of high-fat exercise supplements on gastrointestinal distress, substrate oxidation, and endurance cycling performance. Five female cyclists completed a randomized triple-crossover comprising a two-week diet high in octanoate-rich esterified oil (MCFA), or twice, long-chain fatty acids (LCFA). Following the diets subjects performed 3-h of cycling at 50\% peak power followed by ten maximal sprints while ingesting either a) a carbohydrate (CHO)+MCFA-rich oil emulsion after the two-week MCFA-rich dietary condition (MC-MC, Intervention) and b) after one LCFA-rich dietary condition (LC-MC, Placebo), or c) CHO only following a second LCFA-rich diet (LC$\mathrm{CHO}$, Control). Ingestion of the $\mathrm{CHO}+\mathrm{MCFA}$-rich oil emulsion during 3-h steady-state exercise substantially reduced endogenous-fat oxidation by $88 \%$ (90\% CI: $59-117 \%)$ and $74 \%(40-108 \%)$ in the MC-MC and LC-MC conditions, respectively, relative to LC-CHO, but there was no effect of MCFA adaptation. Adaptation (MC-MC) also had no clear effect on endogenous $\mathrm{CHO}(11 \%,-10-33 \%)$ or exogenous octanoic-acid oxidation rates $(-2 \%$, 20-16\%), relative to the placebo (LC-MC). Ingestion/oxidation efficiency of octanoic acid was high (0.98), but the CHO+MCFA-rich oil emulsion caused mild-moderate gastrointestinal distress during exercise and there was no attenuating effect of MCFA adaptation evident, nor any clear benefit of the oil emulsion or adaptation to performance. Plasma triacylglycerol concentrations were lower by $17 \%(23--4 \%)$ and $15 \%(20--3 \%$; reduction likely) in the LC-MC and MC-MC conditions, respectively, relative to LC-CHO. Total plasma free-fatty acid concentrations were $20 \%$ lower (39-3\%) in the LC-MC condition, relative to LC-CHO. Plasma glycerol concentrations trended lower in the LC-
\end{abstract}


$\mathrm{MC}$ and $\mathrm{MC}-\mathrm{MC}$ conditions, but uncertainty meant these effects were qualified unclear. There was no clear effect of MCFA adaptation on total and ${ }^{13} \mathrm{C}$-enriched plasma octanoicacid concentrations, nor were there any detectable elongation products $\left({ }^{13} \mathrm{C}\right.$-enriched fatty acids $>8: 0$ ) seen in the plasma in either the MC-MC or LC-MC conditions To conclude, despite high efficiency of exogenous-MCFA oxidation, no clear benefit to prolongedendurance performance was observed with the ingestion of a $\mathrm{CHO}+\mathrm{MCFA}$-rich esterified oil emulsion or following adaptation to a MCFA-rich diet in female cyclists.

Key words: medium-chain fatty acids, structured triacylglycerols, dietary adaptation, supplementation 


\section{Introduction}

The finite availability of endogenous glycogen as an energy substrate contributes to the development of fatigue, and is therefore a limiting factor in athletic performance during prolonged strenuous exercise (Kiens and Helge 1998). An increase in plasma free fatty acid (FFA) and fat oxidation has been shown to spare endogenous glycogen, most reliably via fat infusion studies (Vukovich, Costill et al. 1993). However, fat infusion during racing conditions is not feasible and long chain triglyceride ingestion before or during exercise has not been shown to enhance performance (Rowlands and Hopkins 2002). More recently, interest has focused on the ingestion of medium-chain triglycerides (MCTs). Medium-chain fatty acids (MCFA) can be absorbed more rapidly and directly from the intestinal lumen, and their entry into mitochondria does not appear to be rate-limited by the acyl-carnitine transfer system to the extent of long chain fatty acids (LCFA) during high-intensity exercise (Greenberger, Rodgers et al. 1966; Sidossis, Gastaldelli et al. 1997). Therefore, ingestion of MCFAs during exercise has the potential to enhance performance by elevating plasma FFA concentrations and sparing muscle glycogen (Van Zyl, Lambert et al. 1996; VanZyl, Lambert et al. 1996; Vistisen, Nybo et al. 2003).

Previously, studies have focused on the effects of ingesting MCFA-rich exercise supplements on performance in males only. In trials feeding 25-30 g MCT suspensions, the oxidation of MCFA reached $30 \%$ of the ingested quantity compared with $70 \%$ oxidation when co-ingested with carbohydrate (CHO). Plasma FFA and ketone concentrations were elevated, but no overall change in total lipid or CHO oxidation was observed (Jeukendrup, Saris et al. 1995; Jeukendrup, Saris et al. 1996). In contrast, 86 g MCT co-ingested with CHO has resulted in elevation of serum FFA concentration, reduced reliance on 
endogenous $\mathrm{CHO}$, and performance enhancement (2.5\% decrease in $40-\mathrm{km}$ time-trial time) relative to CHO-only ingestion with no reports of gastrointestinal distress (Vistisen, Nybo et al. 2003). Conversely, Jeukendrup et al. (1998) and Goedecke et al. (2005); (1999) did not observe performance benefits when feeding up to $85 \mathrm{~g}$ of MCT co-ingested with $\mathrm{CHO}$ during endurance and ultra-endurance exercise, which was related to the development of moderate-to-severe gastrointestinal distress. Additionally, no clear differences between fat and $\mathrm{CHO}$ oxidation with or without MCT ingestion were observed in these later studies.

Chronic dietary MCT adaptation (Misell, Lagomarcino et al. 2001; Misell, Lagomarcino et al. 2001; Oopik, Timpmann et al. 2001) and ingestion of structured triacylglycerols (Vistisen, Nybo et al. 2003) have been investigated as ways to attenuate gastric distress when ingesting MCTs during exercise. Though gastric distress was alleviated in these studies, no performance benefit was observed when ingesting MCFA supplements during exercise. We recently investigated whether combining a dietary adaptation period (2-weeks) plus ingestion of a structured triacylglycerol (randomizedstructured MCFA oil+CHO) emulsion during exercise would further attenuate gastric distress, and thereby allow a performance benefit to show through due to greater exogenous-energy supply (Thorburn, Vistisen et al. 2006). Gastric distress in response to the MCFA-rich exercise supplement was attenuated during the performance test following the 2-week MCFA adaptation period. In addition, almost 3-fold greater $\left(0.43 \mathrm{~g} \cdot \mathrm{min}^{-1}\right)$ MCFA oxidation was observed in comparison to that previously reported $\left(0.15 \mathrm{~g} \cdot \mathrm{min}^{-1}\right)$ (Jeukendrup, Saris et al. 1996). However, sprint performance remained substantially impaired in comparison to CHO-only. 
The aim of the present study, therefore, was to investigate the effects of a 2 -week MCFA-rich dietary adaptation period and ingestion of MCFA-rich exercise supplements on gastrointestinal distress, MCFA oxidation, and performance in a cohort of trained female cyclist. The majority, but not all, of well controlled studies have shown lower RER in females than males during submaximal endurance exercise (reviewed by (Tarnopolsky 2000) indicating the possibility of proportionately higher fat oxidation. Therefore, females could potentially make better use of exogenous MCFA supplements than their male counterparts, which may translate into performance benefits. 


\section{Materials and Methods}

\section{Subjects}

Five well-trained female cyclists 31 years (SD 3) and $65 \mathrm{~kg}$ (2) completed the study. Three additional subjects withdrew due to illness not related to the study interventions or to other commitments. All cyclists had been training for at least 8-10 h per week and riding competitively for at least 6 months. Mean $\mathrm{VO}_{2} \max$ and peak power output were $3.4 \mathrm{~L} \cdot \mathrm{min}^{-1}$ (0.2) and $243 \mathrm{~W}(9)$ respectively. Before beginning experimentation, all subjects read the study information sheet, were informed of their rights, screened for precluding health conditions, and signed a consent form. The experimental protocol of this study was approved by the Massey University Ethics Committee (Protocol 03/143).

\section{General Design}

Cyclists participated in a randomized double-blind triple-crossover protocol in which three dietary conditions modifying the normal fatty-acid composition were completed over three consecutive 2-week experimental blocks (Figure 1). The conditions were: a) Placebo - 2-week diet containing long-chain fatty-acid rich oil with the intervention CHO+MCFArich esterified oil emulsion ingested during exercise (LC-MC); b) Intervention - 2-week diet containing MCFA-rich esterified oil with the CHO+MCFA-rich oil emulsion ingested during exercise as above (MC-MC); c) Control - 2-week diet containing LCFA-rich oil with a CHO-only exercise supplement (LC-CHO). All subjects began an individualized standardized training regime two weeks before the baseline 3-h ride and performance test (Figure 1). The training was repeated for each subsequent 2-week dietary condition. The training protocol included three-to-five supervised and workload-standardized lab sessions 
where they ingested $500 \mathrm{ml}$ of the dietary supplement during exercise. On the morning of each test, subjects reported to the lab between 6-8 AM in a fasted state, and the parameters of the cycle ergometers were adjusted according to the subject's own racing cycle angles.

Menstrual cycle phase was not controlled. This decision was made because, although suggested, a substantial effect of menstrual cycle phase on carbohydrate and fat metabolism during exercise has not been clearly established (Kanaley, Boileau et al. 1992; Frankovich and Lebrun 2000). Additionally, non-control reduced time that subjects would have to commit to the study from 14 to 8 weeks, thereby increasing the chance of retention, reducing the drift in performance ability, and replicating the conditions employed with males in our lab (Thorburn, Vistisen et al. 2006).

[Insert Figure 1 near here]

\section{Protocols}

Maximal Oxygen Uptake $\left(\mathrm{VO}_{2} \mathrm{max}\right)$ and Peak Power Output (PPO) were measured using a progressive exercise protocol on an electronically-braked cycle ergometer (VeloTron Racer Mate, Seattle, WA). After warm-up, the test started at a workload of 2 $\mathrm{W} \cdot \mathrm{kg}^{-1}$ body mass. The first stage duration was $150 \mathrm{~s}$, after which the load was increased by $50 \mathrm{~W}$, and then by $25 \mathrm{~W}$ for every subsequent 150 -s stage. Exhaustion was defined as the time at which the cyclist could no longer maintain a pedal cadence of 50 RPM after 3 warnings. Maximal oxygen uptake was measured on-line with a calibrated SensorMedics Vmax Spectra Series gas analyzer (SensorMedics Corp., Yorba Linda, CA, USA) and taken as the highest attained 20-s average oxygen uptake. PPO was defined as the last completed 
work rate plus the fraction of time spent in the final non-completed work rate multiplied by the $25 \mathrm{~W}$ work rate increase.

Familiarization. Approximately 1 -week following the $\dot{\mathrm{V}}{ }_{2} \max$ test, a shortened version of the performance test (see below) was performed as a procedural familiarization trial. Subjects cycled for 2-h at 50\% PPO, and performed 6 maximal sprints interspersed with recovery periods at $40 \%$ PPO. An $8 \%$ CHO-based solution was ingested every 20 -min and subjects practiced recording gastrointestinal distress and ratings of relative perceived exertion.

Baseline Test. This test provided information on gastric responses to naïve exposure of the $\mathrm{CHO}+\mathrm{MCFA}$-rich esterified-oil emulsion supplement (see below), doubling as a full practice trial of the testing procedure and a ride to deplete endogenous-glycogen stores to lower background ${ }^{13} \mathrm{C}$ enrichment. Subjects rode for 3 -h at $50 \%$ PPO, primarily for assessment of physiological responses, but also as a long preload/depletion ride prior to the repeated-sprint performance test, which consisted of 10 maximal-effort sprints interspersed with recovery periods as described below.

Immediately before the start of exercise, subjects ingested a double quantity of the CHO+MCFA-rich esterified oil supplement. Every 20-min throughout the 3-h steady-state ride, gastrointestinal distress and exertion ratings were recorded before ingesting the next supplement, the quantity of which was based on individual PPO calculated from a base reference of $220 \mathrm{ml} \cdot 20 \mathrm{~min}^{-1}$ for a PPO of $400 \mathrm{~W}$. Subjects ingested between 127-141 ml of supplement/20 min (254-282 $\mathrm{ml}$ double bolus). During the sprints, subjects ingested each drink supplement throughout the 20 min as they liked, and reported gastrointestinal distress 
and exertion immediately after sprints $1,4,7$ and 10 . Heart rate was continuously recorded by radiotelemetry (S610i Heart Rate Monitor, Polar Electro Oy, Finland).

${ }^{13} \mathrm{C}$ Background Trial. A 3-h ride at 50\% PPO was performed on day 11 of each 2-week supplementation block (Figure 1) to determine background breath ${ }^{13} \mathrm{C}$-enrichment required for later calculation of octanoic-acid oxidation. During the ride, subjects ingested the experimental drink supplement, containing all components except the esterified oil. Every 20-min during the 3 -h ride external respiratory-gas was collected for indirect calorimetry (Sensormedics) and ${ }^{13} \mathrm{C}$ breath enrichment, the latter into $10-\mathrm{ml}$ evacuated rubber-capped test tubes (Labco Ltd., High Wycombe, UK) from a 5-L mixing chamber. For all experiments, the Sensormedics mass flow sensor and gas analyzers were calibrated before testing and every hour during the 3-h exercise test. Following testing, the raw minute volume and gas fractions were adjusted if drift was greater than $3 \%$ following each hour of sampling. Drift between the initial and verification calibrations was assumed to be linear and the raw data were adjusted accordingly.

3-h Ride. On day 14 of each 2-week block subjects repeated the protocol undertaken during the baseline exercise test (Figure 1). Upon reporting to the lab, subjects had a 20GA cannula inserted into the antecubital vein of their right forearm (Becton Dickinson Medical Pte Ltd, Singapore). A 2-way stop-cock valve (Becton Dickinson Medical Pte Ltd, Singapore) was connected to the cannula and a $15-\mathrm{ml}$ blood sample was taken immediately and transferred into vaccutainers (Becton Dickinson \& Co., Franklin Lakes, USA) containing lithium heparin $(5 \mathrm{ml})$ and EDTA $(10 \mathrm{ml})$. Triplicate breath samples were collected from the mixing chamber for resting breath ${ }^{13} \mathrm{C}$ enrichment. Immediately before exercise began, subjects consumed a double bolus of drink supplement. Every 20-min during the 3-h ride data variables were collected in the order of exertion and gastrointestinal 
distress scales, indirect calorimetry, ${ }^{13} \mathrm{C}$ breath enrichment, and a blood sample. Immediately following data collection another bolus of supplement was ingested. Heart rate was continuously recorded.

Performance Test. After completion of the 3-h ride, subjects dismounted their cycles and were allowed to toilet and stretch. Upon remounting their ergometers, subjects completed ten maximal sprints interspersed and beginning with a recovery interval at $40 \%$ PPO. The duration of the sprint (2-3 $\mathrm{min})$ and recovery (5-6 min) periods were based on work done (kilocalories), determined by individual PPO (0.125 x PPO). Fixed linear workloads approximately equivalent to riding a 39 or 56 front chain ring and a 10 -spocket 21 to 11 tooth rear cluster were programmed into the Velotron software. A gear switch was positioned on the end of the right handlebar break hood to provide convenient changing of linear resistance. Cyclists self-selected cadence and gearing but were instructed to sprint as fast as possible until the required kilocalories were achieved. No verbal encouragement was provided to the subjects, the only information provided during the sprints was elapsed work completed (kilocalories) shown on the computer screen. Subjects were given a verbal count down in preparation for the start of each sprint and at 20,10, 5 and 2 kilocalories to go in preparation for the end of each sprint.

Supplement ingestion continued during the sprint procedure with the allocated quantity ingested ad libitum every $20 \mathrm{~min}$. Immediately after sprints 1, 4, 7 and 10, exertion and gastrointestinal distress data were collected. A final $10 \mathrm{ml}$ blood sample was taken immediately upon completion of the final sprint. 


\section{Diet and Supplements}

Diet and Dietary Supplementation. Immediately following the baseline test subjects started their first 2-week diet condition. The diets consisted of food and drink supplements containing either the MCFA-rich ( $1 / 3$ canola and $2 / 3$ trioctanoin esterified oils) or LCFA (canola only) oils in prescribed meal and snack replacements. Both MCFA and LCFA diets were administered in the same way so as to blind the subjects to each condition. Foods and supplements were prepared on site by food technologists and catering services and included muffins, sports fudge bars, curries, bolognese and milk-like drinks. Each item was produced to provide $15 \mathrm{~g}$ of experimental oil (either LCFA or MCFA-rich) per serving and a fixed macronutrient composition: 15, 55, and 30 percent energy from protein, carbohydrate, and oil, respectively, so that fortnightly consumption of the supplements and other foods could be replicated. The amount of food and supplements ingested by each subject was determined by body mass, relative to a model $65-\mathrm{kg}$ female cyclist ingesting 78 $\mathrm{g}$ of the experimental oils per day. In practice, the quantity of randomized esterified oil ingested during the 2-week diet (intervention condition) was $81 \mathrm{~g} \cdot \mathrm{d}^{-1}$ (SD 6) $\left(54 \mathrm{~g} \mathrm{MCFA} \cdot \mathrm{d}^{-}\right.$ ${ }^{1}$ SD 4). Subjects recorded all food ingested over the first two-week experimental block and replicated that daily pattern over the proceeding two blocks. In addition to eating the foods and supplements during the day, subjects were instructed to ingest the sports bars and milklike drinks during training sessions to facilitate exercise gastrointestinal adaptation. Subjects were also provided with extensive lists and instructed not to eat any foods naturally enriched in ${ }^{13} \mathrm{C}$ (cane sugar and maize products) beginning immediately after the baseline test, but to otherwise maintain their normal diet. Instructing subjects in this way has been shown to be effective in reducing background ${ }^{13} \mathrm{C}$ enrichment (Jeukendrup, Saris 
et al. 1995; Jeukendrup, Saris et al. 1995; Jeukendrup, Saris et al. 1996). Subjects verbally reported adhering to the dietary interventions. Two subjects reported experiencing slight cases of diarrhea and belching while on the intervention (MC-MC) diet though none claimed to be aware of which condition they were on at the time. Both the LCFA- and MCFA-rich diets were otherwise well tolerated.

Exercise Supplements. Four different milk-like drinks and emulsions were formulated for use during exercise. The drinks were flavored identically for placebo purposes. The drink containing the CHO+MCFA-rich esterified oil was made in two forms: the supplement ingested during the baseline, LC-MC and MC-MC performance (sprints) tests contained $5.7 \%$ esterified oil, $2.6 \%$ wheat-derived dextrose and $5.6 \%$ maltodextrin, $1.5 \%$ sodium caseinate emulsifier, $0.08 \%$ salt, colors, chocolate and vanilla flavoring; an identical supplement was ingested during the 3-h ride in the MC-MC and LC-MC dietary conditions, but also contained $1-{ }^{13} \mathrm{C}$ octanoic-acid tracer incorporated into the esterified oil. The third, placebo-control exercise drink used in the LC-CHO condition contained the same ingredients as above minus the esterified oil, and with addition of $4.0 \%$ milk powder to emulate color and taste. The fourth drink used during the three ${ }^{13} \mathrm{C}$ background trials consisted of identical ingredients to the drink containing the MCFA-rich esterified oil minus the oil and ${ }^{13} \mathrm{C}$ tracer. The mean quantities of MCFA-rich esterified oil emulsion ingested were $77 \mathrm{~g}$ (SD 3) (51 g MCFA SD 2) and $31 \mathrm{~g}$ (SD 1.2) (21 g MCFA, SD 0.8) during the 3 -h ride and performance test, respectively.

In producing the exercise supplements, the oil was first heated to $50^{\circ} \mathrm{C}$. Dry ingredients were mixed with water, colors and flavors, and the two mixtures were then combined and homogenized to disperse the fat droplets (giving the drinks a milky appearance) and 
refrigerated. The drinks were made 1-5 days before ingestion and had a minimum refrigerated shelf life of 10 days.

\section{Randomized-Esterified Oil}

A blended oil mixture consisting of $1 / 3$ canola (LCT) and $2 / 3$ trioctanoin oil was mixed in a batch reactor by an impeller at $230 \mathrm{rpm}$. After the vacuum reached $100 \mathrm{mbar}$, the oil was dried for $30 \mathrm{~min}$ at $90^{\circ} \mathrm{C}$. Temperature was then decreased to $60^{\circ} \mathrm{C}$ and $0.1 \mathrm{wt} \%$ sodium methoxide was added while stirring. Any air in the reactor was removed using vacuum and $\mathrm{N}_{2}$ systems. After $30 \mathrm{~min}$, the reaction was stopped by addition of a $5 \mathrm{wt} \%$ citric-acid water solution. The oil was washed 3-4 times until $\mathrm{pH}$ was below 7, and dried again before deodorization in a conventional batch deodorizer. Vacuum was adjusted to less than 5 mbar, stripping steam consumption was adjusted to $4 \mathrm{wt} \%$, and temperature was raised to $160^{\circ} \mathrm{C}$ for $2 \mathrm{~h}$. After deodorization, the oil was cooled by tap water circulation with $\mathrm{N}_{2}$ protection, and stored in a $-25^{\circ} \mathrm{C}$ freezer.

\section{$1-{ }^{13}$ C-Octanoic Acid-Enriched Esterified Oil}

The $1-{ }^{13} \mathrm{C}$-octanoic acid-enriched esterified oil was produced by esterification of ${ }^{13} \mathrm{C}$ labelled octanoic acid (C8:0) (Cambridge Isotope Laboratories, Andover, MA), oleic acid $(18: 1 n-9)$ and glycerol in the proportion of 3:1:1.6. Components were mixed 10:1 with lipase (Novozym, Novozymes, Bagsvaerd, Denmark) at $60^{\circ} \mathrm{C}$ with constant stirring. Vacuum removed excess water from the process by passing the air through anhydrous $\mathrm{Na}_{2} \mathrm{SO}_{4}$ (JT Baker, Deventer, Holland). After a $24 \mathrm{~h}$ incubation period, the reaction was stopped by separating the oil and enzymes with filtering. The oil was then kept at $-20^{\circ} \mathrm{C}$ 
until use. The ${ }^{13} \mathrm{C}$-enriched oil was mixed into the randomized esterified oil that yielded a mean oil ${ }^{13} \mathrm{C}$-enrichment of $84 \delta \cdot \mathrm{mil}^{-1}$ vs. PDB (SD 30) $\left(0.006854{ }^{13} \mathrm{C} /{ }^{12} \mathrm{C}\right.$ ratio).

\section{Psychometric Scales}

Perceived exertion (soreness of legs and effort of cycling) and gastrointestinal distress (nausea, fullness/bloatedness, stomach cramp, reflux/burping) markers were measured using scales modeled from Borg's CR10 scale (Borg et al. 1998). Verbal descriptors were associated with the scale: nothing 0 , very weak 1 , weak or mild 2 , moderate 3 , strong 5 , very strong 7 , extremely strong 10, and absolute maximum 13.5. Subjects were instructed to make a pen mark on a continuous scale, rating the strength of their exertion or distress.

\section{Analyses}

${ }^{13} \mathrm{C}$ Enrichment. Breath samples were analyzed for ${ }^{13} \mathrm{C} /{ }^{12} \mathrm{C}$ by gas chromatography continuous flow isotope ratio mass spectrometry (Finnigan Delta XP, Bremen, Germany). The enrichment of the esterified oil was measured using Elemental Analyzer isotope ratio mass spectrometry.

Calculations. Oxidation rates $\left(\mathrm{g} \cdot \mathrm{min}^{-1}\right)$ of exogenous octanoic acid and total fat and $\mathrm{CHO}$ were calculated from ${ }^{13} \mathrm{C}$-enrichment and indirect calorimetry measurements. Isotopic enrichment of expired air was expressed as the delta per million difference $(\delta \%)$ between ${ }^{13} \mathrm{C} /{ }^{12} \mathrm{C}$ ratio of the sample and a known laboratory reference standard (Pee Dee Belemnite; PDB) according to the formula: $\delta^{13} \mathrm{C}=\left[\left({ }^{13} \mathrm{C} /{ }^{12} \mathrm{C}\right.\right.$ ratio sample $/{ }^{13} \mathrm{C} /{ }^{12} \mathrm{C}$ ratio standard $)-$ $1] \cdot 10^{3} \%$, where, ${ }^{13} \mathrm{C} /{ }^{12} \mathrm{C}$ standard $=0.0112372$ (Craig 1957). The amount of octanoic-acid oxidized is then calculated according to the formula: Exogenous octanoic-acid oxidation $\left(\mathrm{g} \cdot \min ^{-1}\right)=\mathrm{VCO}_{2} \cdot\left(\delta_{\mathrm{Exp}}-\delta_{\mathrm{bkg}} / \delta_{\text {Ing }}-\delta_{\mathrm{bkg}}\right) \cdot(1 / k)$, in which $\delta_{\mathrm{bkg}}$ is the ${ }^{13} \mathrm{C}$ enrichment of expired 
air in the 3-h background trial, $\delta_{\operatorname{Exp}}$ is the ${ }^{13} \mathrm{C}$ enrichment of expired air during the 3 -h ride with ${ }^{13} \mathrm{C}$-enriched esterified oil ingestion, $\delta_{\text {Ing }}$ is the ${ }^{13} \mathrm{C}$ enrichment of the oil in the ingested exercise supplement, and $\mathrm{k}$ is the amount of $\mathrm{CO}_{2}$ (liters) produced via oxidation of $1 \mathrm{~g}$ octanoic acid on a glycerol backbone $\left(\mathrm{k}=1.2369 \mathrm{~L} \mathrm{CO}_{2} \cdot \mathrm{g}_{\text {trioctanoin }}{ }^{-1}\right)($ Jeukendrup, Saris et al. 1995). A conversion factor of $34.19 \mathrm{~kJ}^{-1}{ }^{-1}$ was used to estimate MCFA contribution to energy expenditure (Livesey and Elia 1988).

Total carbohydrate and fat-oxidation rates were calculated using the non-protein respiratory quotient (Jeukendrup and Wallis 2005): Carbohydrate oxidation $\left(\mathrm{g} \cdot \mathrm{min}^{-1}\right)=$ 4.210 $\cdot \mathrm{VCO}_{2}-2.962 \cdot \mathrm{VO}_{2}$, Fat oxidation $\left(\mathrm{g} \cdot \mathrm{min}^{-1}\right)=1.695 \cdot \mathrm{VO}_{2}-1.701 \cdot \mathrm{VCO}_{2}$. Conversion factors of $15.64 \mathrm{~kJ} \cdot \mathrm{g}^{-1}$ (Ferrannini 1988) for $\mathrm{CHO}$, and $40.81 \mathrm{~kJ} \cdot \mathrm{g}^{-1}$ (Péronnet and Massicotte 1991) for fat oxidation were used to estimate contribution to energy expenditure. The oxidation rate of other fats was the total fat-oxidation rate minus the octanoic acid oxidation rate.

Calculation of exogenous substrate oxidation is affected by the delayed equilibration of ${ }^{13} \mathrm{CO}_{2}$ originating from the tissues with the large endogenous $\mathrm{HCO}_{3}{ }^{-}$pool. However, a physiological steady-state condition occurs relatively rapidly during exercise, and ${ }^{13} \mathrm{CO}_{2}$ in the expired air will be equilibrated with the ${ }^{13} \mathrm{CO}_{2} / \mathrm{H}^{13} \mathrm{CO}_{2}$ pool from around 60 min of exercise (Robert 1987). As a consequence, calculations on substrate oxidation were only reported from 60 to 180 min of exercise.

Plasma. Blood was collected into lithium heparin and EDTA-containing tubes, immediately centrifuged at $2000 \mathrm{G}$ for $12 \mathrm{~min}$, aspirated into eppendorf tubes and snapfrozen in liquid nitrogen before being stored in a $-80^{\circ} \mathrm{C}$ freezer until analysis. Plasma lactate, glucose, potassium, and acid-base variables were analyzed using an automated 
blood gas analyzer (Bayer Rapidlab 800 system, Bayer HealthCare LLC, Tarrytown, NY, U.S.A). Before analysis, a quality control test was run to evaluate the system for imprecision and inaccuracy. Two-point calibrations for all parameters were performed every 45-60 samples. One-point calibrations for all parameters were performed every 15-20 samples. One-point calibrations for $\mathrm{PCO}_{2}$, glucose and lactate were performed every 3 samples.

Triacylglycerol and glycerol concentrations were determined by thin layer and gas chromatography as described previously (Vistisen, Nybo et al. 2003). Extraction of plasma fatty acid for the determination of fatty acid concentration and isotopic enrichment was performed according to Patterson and colleagues (Patterson, Zhao et al. 1999). Fatty acid concentration was measured by gas chromatography with FID (Autosystem XL; Perkin Elmer, Northwalk, CT, USA), using 9:0 and 17:0 as internal standards. Plasma fatty acid ${ }^{13} \mathrm{C}$ enrichment was measured by gas chromatography-combustion isotope ratio mass spectrometry (GC-C-IRMS, Hewlett Packard 5890, Finnigan GC combustion III, Finnigan Deltaplus; Finnigan MAT, Bremen, Germany) as described previously (Van Hall, Bulow et al. 2002). 8:0 enrichment was corrected by a factor of 9/8 to account for the extra methyl group of the methyl octanoate derivative. A standard sample (Nu-Chek Prep, Elysian, MN) with known composition and quantities of fatty acid methyl esters was run daily. This standard contained fatty acid methyl esters with chain length of C6 to C22, which ensured detection of all plasma fatty acid methyl esters within this range. Fatty acids were identified by retention times of the standard fatty acid methyl esters.

Sprint Data. Mean power $\left(\mathrm{J} \cdot \mathrm{s}^{-1}\right)$ for each interval was calculated from the inbuilt efficiency factor and $\mathrm{J}$ conversion factors in the Velotron software code: kcal·4.186 $\cdot 1000 \cdot 0.25 / \mathrm{t}$, where $\mathrm{kcal}$ is the energy (kilocalories) expended by the subject during the 
sprint, 4.186 is the conversion factor from kilocalories to kilojoules, 0.25 is the efficiency factor of an exercising person, and $t$ is the time it took the subject to complete the sprint in seconds.

\section{Statistics}

General Method. The effect of diet condition on the outcome variables was estimated with mixed modeling in the Statistical Analysis System (SAS9.1, SAS Institute, Cary, NC). Most dependent variables were analyzed after log transformation to reduce the effects of non-uniformity of error. For measures of performance, metabolism, and other dependent physiological variables, the effects of the diets were compared in a three-way model, whereas the analysis of psychometric variables also included the Baseline test responses. Quantitative mixed linear models were applied to the time-series data sets for the 3-h exercise and during the sprint procedure where sample time or sprint number were numeric effects. For all datasets treatment was a fixed effect and for the performance and psychometric analyses an order term was included. In all data sets, the random effects were subject and the interaction between subject and condition. The random variability associated with moving between sprints (fatigue) or progression along a time series was also included in the analysis for the relevant data sets. The within-cyclist standard deviation was estimated from the residual variance. A mechanisms analysis of the relationship between power output and nausea was conducted as described previously (Thorburn, Vistisen et al. 2006).

Presentation of Data. Measures of centrality and spread for subject descriptive, raw stable isotope, and dietary variables are raw means and standard deviations (SD). Means derived from the analysis of log-transformed variables are back log-transformed least- 
squares, or adjusted, means. The associated spread around these least-squares mean is represented by percentage (geometric) standard deviations or factor standard deviations $\left(\mathrm{SD}_{\mathrm{f}}\right)$, implying $\times / \div$. For example, for a plasma-glucose concentration of $5 \mathrm{mmol} \cdot \mathrm{L}^{-1}$ with a between-subject standard deviation of $20 \%\left(\mathrm{SD}_{\mathrm{f}} 20\right)$, the typical variation is $5 \times 1.20$ to $5 \div$ 1.20 , or 6 to $4.16 \mathrm{mmol} \cdot \mathrm{L}^{-1}$. Data in graphs and text are shown as least-squares means. Data are rounded to two significant digits or in some cases three where the first digit is " 1 ".

Precision of the Estimate and Practical Inference. In keeping with recent trends in inferential statistics, e.g., (Curran-Everett and Benos 2004; Sterne and Smith 2001), we made quantitative inferences about population (true) effect by expressing uncertainty as $90 \%$ confidence limits $(\mathrm{CL})$ or interval $(\mathrm{CI})$ and as probabilities that the true value represents a practically-important substantial change (beneficial or detrimental, increase or decrease) (Batterham and Hopkins 2006). The smallest substantive change in sprint power was $1.1 \%$ as previously (Thorburn, Vistisen et al. 2006), with females assumed to be similar (Paton and Hopkins 2005). The smallest standardized (Cohen) change (i.e. effectsize statistic) in the mean for biochemical and psychometric variables was 0.20 times the between-subject SD for the value in the control group (LC-CHO condition) (Cohen 1988). Probabilities of a substantial benefit or detriment, increase or decrease were calculated for 90\% CL from the two-tailed Student t-distribution (Hopkins 2001) and inferred as follows: $<1 \%$, almost certainly not; $1-5 \%$, very unlikely; $5-25 \%$, unlikely; $25-75 \%$, possible; $75-$ 95\%, likely; 95-99\%, very likely; >99\%, almost certain (Batterham and Hopkins 2006). An effect was qualified unclear (not clear, inconclusive) if its confidence interval overlapped both thresholds for substantiveness by more than $5 \%$. In the case where the chance that the 
benefit or detriment is $<5 \%$ and the chance of the effect being trivial is greater than a substantial benefit or detriment, the trivial likelihood is qualified. 


\section{Results}

\section{Performance}

No clear effect of diet on mean power or fatigue (slope) was observed between the three supplemental conditions (Figure 2, Table 1).

\section{Mechanisms Analysis}

For every 1 unit increase in the position of the nausea curve, the position of the mean power curve decreased by $0.4 \mathrm{~W}(90 \% \mathrm{CI}$ : $-3.6-4.4 \mathrm{~W}$; effect unclear). Nausea had no detectable influence on sprint mean power when added as a covariate in the polynomial analysis (Figure 3).

\section{Substrate Metabolism}

Breath ${ }^{13} \mathrm{C}$-enrichments during the 3-h background trials are shown in Figure 4. Mean peak octanoic-acid oxidation rates at the 180-min sample during the LC-MC and MC-MC conditions, respectively, were $0.31 \mathrm{~g} \cdot \mathrm{min}^{-1}\left(\mathrm{SD}_{\mathrm{f}} 32 \%\right)$ and $0.32 \mathrm{~g} \cdot \mathrm{min}^{-1}(32 \%)$. There was no clear effect of MCFA adaptation (MC-MC condition) on exogenous octanoic-acid oxidation (vs. LC-MC), but interestingly, the oxidation efficiency (ingestion rate/oxidation rate) over the period $60-180$ min was $98 \%$ in the LC-MC condition and $99 \%$ in the MC-MC condition.

Overall relative energy contributions are shown in Figure 5. Energy derived from the oxidation of endogenous fat was substantially reduced by the oil emulsion (MC-MC: -88; 59-117\%, decrease almost certain; LC-MC -74; -108- -40\%, decrease almost certain) conditions relative to $\mathrm{LC}-\mathrm{CHO}$, but there was no effect of MCFA adaptation evident. There 
was also a likely reduction $(-14 \% ;-29-1 \%)$ in energy derived from endogenous-CHO oxidation in the LC-MC condition relative to LC-CHO, but the differences between the other comparisons were unclear.

The pattern of substrate oxidation over the $3-\mathrm{h}$ ride is illustrated in Figure 6. The increase in the octanoic-acid oxidation rate from the $60^{\text {th }}$ to the $180^{\text {th }}$ min sample points was $0.09 \mathrm{~g} \cdot \mathrm{min}^{-1}$ in the LC-MC condition and $0.10 \mathrm{~g} \cdot \mathrm{min}^{-1}$ in the MC-MC condition, but consistent with the overall average response, there was no clear difference between conditions. Total $\mathrm{CHO}$ oxidation rates changed little $(<10 \%)$ from 60 to $180 \mathrm{~min}$; in contrast endogenous fat-oxidation rates declined by 0.04 and $0.11 \mathrm{~g} \cdot \mathrm{min}^{-1}$ in the LC-MC and MC-MC, respectively, with only a $0.01 \mathrm{~g} \cdot \mathrm{min}^{-1}$ decline in the LC-CHO condition. The difference in the decline in MC-MC relative to $\mathrm{LC}-\mathrm{CHO}$ was 3.2-fold (0.7-14). The remaining observed between-treatment slope differences for endogenous-fat oxidation were inconclusive.

\section{Psychometric Scales}

A statistical summary of gastrointestinal distress (nausea, fullness/bloating, stomach cramp, reflux) and exertional parameters (leg soreness, perceived effort) is shown in Table 2. The responses for nausea, fullness/bloatedness, and perceived effort were selected to illustrate the magnitude and temporal effects of treatment and exercise duration (Figure 7).

3-h ride. The treatment effects on gastrointestinal distress and exertion were similar, but the effect of the MC-MC condition relative to LC-CHO on fullness/bloating, stomach cramp, and leg soreness were unclear (Table 2). There were no clear consistent patterns in slope (increase in scale unit rating) from the $20^{\text {th }}$ min to the $180^{\text {th }}$ min sample points. 
Performance test. The performance test accentuated the effect of the LC-MC treatment on nausea and fullness/bloating, relative to the LC-CHO condition. The exertional parameters were also substantially increased in the LC-MC condition but not clearly in the MC-MC condition, relative to the LC-CHO control. The effect of the MC-MC condition on gastrointestinal distress markers relative to LC-MC was unclear. There were no clear consistent slope patterns from sprints 1 to 10 (not shown).

\section{Plasma, Electrolytes, Acid-base Status, Glucose and Lactate}

No clear differences were observed between conditions for hydrogen ions or standard bicarbonate concentrations. Glucose was 5.2\% (1.5-8.9\%) lower in the MC-MC condition, relative to $\mathrm{LC}-\mathrm{CHO}$; no clear effect was observed between LC-MC and LC-CHO or LCMC and MC-MC conditions. Lactate was 7.9\% (1.6-14.2\%) lower in the LC-MC condition, relative to $\mathrm{LC}-\mathrm{CHO}$; no clear effect was observed between $\mathrm{MC}-\mathrm{MC}$ and LC$\mathrm{CHO}$ or MC-MC and LC-MC conditions. The outcomes for the remaining electrolyte comparisons were qualified as possible increases or decreases (25-75\% likelihood) or unclear.

\section{Plasma Lipids}

The effects of treatment condition on plasma lipids and octanoic-acid ${ }^{13} \mathrm{C}$ enrichment are shown in Figure 8. At rest and during the 3-h steady-state ride plasma triacylglycerol concentrations were overall lower by $17 \%(23--4 \%$; reduction likely) and $15 \%(20--3 \%$; reduction likely) in the LC-MC and MC-MC conditions, respectively, relative to LC-CHO; there was no clear effect of exercise, however. Plasma glycerol concentrations trended 
overall lower by $25 \%(54--18 \%)$ and $45 \%(84--17 \%)$ in the LC-MC and MC-MC conditions, respectively relative to $\mathrm{LC}-\mathrm{CHO}$, but the uncertainty meant these effects were qualified unclear. At the end of the sprints, there was a $50 \%$ lower $(137--6 \%$; likely reduction) plasma glycerol concentration. Total plasma free fatty acids were also $27 \%$ (73$-7 \%$; unclear) and 20\% (39-3\%; reduction likely) lower in the LC-MC and MC-MC conditions, respectively, relative to LC-CHO. There was no effect of MCFA adaptation on total and ${ }^{13} \mathrm{C}$-enriched plasma octanoic-acid concentrations. There were no detectable elongation products $\left({ }^{13} \mathrm{C}\right.$-enriched fatty acids $\left.>8: 0\right)$ seen in the plasma in either the MC-MC or LC-MC condition (data not shown). All remaining comparisons for the respective variables were unclear. 


\section{Discussion}

The purpose of this study was to determine if a 2-week high-MCFA dietary adaptation period would attenuate gastrointestinal distress, increase octanoic-acid oxidation rate, and enhance performance while ingesting a $\mathrm{CHO}+\mathrm{MCFA}$-rich oil emulsion during endurance cycling in a sample of well-trained female cyclists. The CHO+MCFA-rich oil emulsion resulted in mild-to-moderate gastrointestinal distress ratings, relative to the LC-CHO condition, and there was no evidence for any attenuation with dietary-MCFA adaptation. High amounts of ingested MCFA were oxidized during the 3-h pre-load exercise, and a substantial reduction in endogenous fat availability and metabolism was observed with MCFA-rich oil emulsion ingestion. However, there was no clear effect of MCFA adaptation on octanoic-acid oxidation, ingested ${ }^{13} \mathrm{C}$-octanoic acid and endogenous carbohydrate metabolism, or endurance cycling performance.

The purpose of the 2-week MCFA-dietary adaptation intervention was to attempt to alleviate the gastrointestinal distress normally associated with high MCFA ingestion rates during exercise and, therefore, to observe any potential benefit to substrate supply and high-intensity endurance performance. In male subjects, we (Thorburn, Vistisen et al. 2006) previously observed a clear substantial reduction in gastrointestinal distress parameters with MCFA adaptation; and in the covariate analysis, nausea had a moderate-strong reducing effect on sprint mean power. Conversely, in the current cohort of female subjects MCFA adaptation did not clearly attenuate gastrointestinal distress symptoms, and performance was not adversely affected by the intervention relative to the smallest worthwhile detriment in mean power (Table 1), or in relation to the distress marker nausea (Figure 3). However, 
only five female subjects completed the present study leading to modest power and contributing to the uncertainty. The confidence limits for mean sprint power suggest that, relative to the $\mathrm{LC}-\mathrm{CHO}$ condition, the true mean population effect of MCFA adaptation (MC-MC condition) on performance could be a substantial benefit of up to $2.8 \%$, or an impairment in the order of 5.0\%; for the control LC-MC condition the true effect could be an enhancement of up to $6.6 \%$, or impairment of $10 \%$. This uncertainty in the direction of the true population response implicates individual differences in response to the treatment, or random error. The sample size required to make a confident statement about whether the true population mean effect for the MC-MC vs. LC-CHO comparison is trivial $(\leq 1.1 \%$ impairment or enhancement) or substantially negative is infinite, because the observed effect (1.1\% impairment, Table 1$)$ is the same as the smallest worthwhile effect $(1.1 \%)$, so no matter how many subjects are tested, there will be $50 \%$ probability that the true population mean will be both substantially negative or trivial. For the LC-MC vs LC-CHO comparison, however, the sample size to conclude a very likely substantial detriment is 17 (n required for clear-cut substantial positive or negative effect $=($ SQRT(width or observed CI/width of acceptable CI)) $\times$ existing n; source: W. Hopkins, 2006 unpublished). Therefore, were we able to have completed testing on all 8 subjects, or extended the cohort to 10-12 - a large sample for these types of studies - our conclusions may have been little different.

Meanwhile, the finding of an attenuated response to the intervention relative to the male cohort studied previously (Thorburn, Vistisen et al. 2006) is consistent with the generalization that trained females respond with lower amplitude to nutritional-exercise 
interventions, relative to their male counterparts. Compared with males, the observed average reductions in sprint performance and endogenous-fat oxidation with naïve exposure to the MCFA-CHO exercise supplement (LC-CHO condition) were less in magnitude, and following MCFA adaptation (MC-MC condition) the attenuating effects of octanoic acid and gastrointestinal distress markers were unclear in the females. Other's have found that trained females respond in a lesser magnitude to a number of nutritional interventions including recovery in response to high-protein recovery supplements (D.S. Rowlands, 2005 unpublished), carbohydrate loading, and creatine supplementation (Tarnopolsky 2000).

All previous published work examining the ergogenic potential and metabolic effects of MCT-CHO ingestion during exercise has been conducted in well-trained males with the outcomes equivocal. In response to naïve exposure, Goedecke et al. (2005) found $\mathrm{CHO}+\mathrm{MCT}$ ingestion to clearly impair performance relative to $\mathrm{CHO}$-only during $\sim 5$-h of exercise. In their study, the performance of intermediary sprints became increasingly worse, and time to complete the final $200-\mathrm{kJ}$ time trial was longer by $13 \%$ (90\%CL: 8 to $18 \%$ ) with $\mathrm{CHO}+\mathrm{MCT}$ ingestion. In an earlier study, Goedecke et al. (1999) observed mean impairments of $3 \%$ and $2.5 \%$ in $40-\mathrm{km}$ time-trial performance following a 2 -h ride reported as statistically insignificant (P-values not provided) when subjects ingested high $\mathrm{MCT}+\mathrm{CHO}$ and low $\mathrm{MCT}+\mathrm{CHO}$ supplements, respectively, in comparison to $\mathrm{CHO}$-only. On the other hand, other authors have reported non-statistically clear (P-value $>0.05)$ trends toward performance improvements with $\mathrm{CHO}+\mathrm{MCT}$ ingestion. Jeukendrup et al. (1998) and Vistisen et al. (2003) observed on average 1\% and 1.5\% improvements in constant work or constant time tests, respectively, when subjects ingested a CHO+MCT supplement in comparison to $\mathrm{CHO}-$ only. The only group reporting a clear performance benefit as a 
2.5\% (90\%L: 0.5-4.5) enhancement in 40-km time following a 2-h pre-load was also the only group reporting no gastrointestinal distress (Van Zyl, Lambert et al. 1996). This finding is in contrast to all of the other studies feeding $>30 \mathrm{~g}$ of MCT, including our earlier adaptation study (Thorburn, Vistisen et al. 2006). Van Zyl et al. (1996) suggested that the increase in performance in their study was due to the observed increased availability of plasma fatty acids and the subsequent sparing of endogenous carbohydrate (implying muscle glycogen). This result contrasts the present findings, as well as that of others (Goedecke, Elmer-English et al. 1999; Jeukendrup, Saris et al. 1995; Jeukendrup, Thielen et al. 1998; Thorburn, Vistisen et al. 2006) which show that MCT ingestion during exercise spares only endogenous fat rather than endogenous carbohydrate utilization. In addition, the present study showed a possible reduction in plasma lipid (triacylglycerol, glycerol and total fatty acid) availability with MCFA ingestion in comparison to the LC-CHO condition, suggesting reduced rather than increased plasma fatty-acid availability (Figure 8).

That there was no clear evidence supporting a performance benefit in this and several previous studies might be attributed to the substantial gastrointestinal distress (Jeukendrup, Thielen et al. 1998; Goedecke, Elmer-English et al. 1999; Goedecke, Clark et al. 2005; Thorburn, Vistisen et al. 2006); indeed we previously provided evidence through quantifying the relationship with nausea (Thorburn, Vistisen et al. 2006), though in the present study we found no evidence for a relationship between gastrointestinal distress and impaired performance. We previously suggested that MCFAs may not even reach the systemic circulation as MCFAs, which would exclude utilization of their potentially beneficial properties in the muscle cells (Vistisen, Nybo et al. 2003; Thorburn, Vistisen et al. 2006). Fatty-acid elongation or storage in the liver prior to release into the circulation, 
conversion to ketone bodies which were then later oxidized or excreted through the urine or breath, or malabsorption by the gut and excretion in the feces we proposed as possible fates. However, several observations in the present study suggest that the muscle was the more likely site for MCFA disposal during exercise: we observed ${ }^{13} \mathrm{C}$-octanoic acid in the plasma and the oxidation rates were rapid and exclusive; there were no measured elongation products; and the average energy provided by octanoic-acid oxidation was approximately matched by the reduction in energy provided from endogenous-fat oxidation (Figure 5).

Although we can not be certain that all of the ingested MCFAs were metabolised by the exercising muscle, we did make some further interesting and novel observations pertaining to the $\mathrm{CHO}+\mathrm{MCFA}$-rich esterified oil emulsion. In males, we found a high efficiency of octanoic-acid oxidation of up to $\sim 100 \%$ ingestion rate $\left(0.43 \mathrm{~g} \cdot \mathrm{min}^{-1}, 5.3 \mathrm{mg} \cdot \mathrm{kg}^{-1} \cdot \mathrm{min}^{-1}\right)$ when co-ingested with $\mathrm{CHO}$ (Thorburn, Vistisen et al. 2006). In the present study the maximum octanoic-acid oxidation rate reached in the $180^{\text {th }}$ minute of the 3 -h ride was similar (4.9 $\left.\mathrm{mg} \cdot \mathrm{kg}^{-1} \cdot \mathrm{min}^{-1}\right)$, which also approximated the MCFA ingestion rate (Figure 6). These oxidation rates are 2-3 fold higher than previously reported $\left(0.15 \mathrm{~g} \cdot \mathrm{min}^{-1}\right.$, (Jeukendrup, Saris et al. 1996) and suggest a unique quality of the exercise supplements promoting high absorption and oxidation rates. Fat digestion is normally slow because triacylglycerols aggregate into large droplets in the small intestine, slowing the action of pancreatic lipase. However, our supplements were suspended in an emulsion, which distributes lipids into smaller droplets, thereby increasing their accessibility to pancreatic lipase. Also, cyclists were fed MCFAs and long chain fatty acids in the form of structured triacylglycerols, which may have enhanced MCFA absorption due to the increase in 
pancreatic secretion which is stimulated by long-chain triglyceride, but not MCT ingestion (Mott, Sarles et al. 1972). Therefore, more extensive hydrolysis of the structured triacylglycerols may have occurred in comparison to MCT solution/suspensions used in previous MCFA-oxidation rate studies (Jeukendrup, Saris et al. 1996), leading to greater absorption, blood concentrations, and mitochondrial oxidation. Despite the higher oxidation rates, there was little evidence for an effect of MCFA adaptation on absorption or metabolic clearance rates based on the observation of similar plasma ${ }^{13} \mathrm{C}$-enriched octanoic-acid concentrations, relative to the LC-MC control.

In summary, the ingestion of a CHO+MCFA-rich esterified oil emulsion resulted in high efficiency of exogenous MCFA oxidation, but induced mild-moderate gastric distress symptoms during prolonged exercise in female cyclists. Two weeks' adaptation to a MCFA-rich diet did not attenuate the gastrointestinal distress symptoms, and no clear performance benefit was observed with ingestion of the $\mathrm{CHO}+\mathrm{MCFA}$-rich exercise supplement or following adaptation. Similar to the consensus from previous adaptation and naïve exposure studies in males, there was little evidence to suggest that increasing exogenous energy supply through the addition of MCFAs to the training diet or exercise supplements will result in ergogenic outcomes in trained female cyclists. While some uncertainty remains, the results of our investigations lead to the conclusion that further investigation of MCFA ingestion as a potential ergogenic aid for exercise performance is not warranted. 


\section{Acknowledgements}

The subjects for their contributions to make this project possible. Agnes Gauliard for technical assistance. Harvey Bourne and George Thorburn for the dietary supplements. Will Hopkins for advice on mixed modeling. The study was funded by Massey University Wellington Strategic Grant PR56953-1207 and by The Danish Medical Research Council (Grant 22-04-0596). 


\section{References}

Batterham, A. M. and W. G. Hopkins (2006). Making meaningful inferences about magnitudes. International Journal of Sport Physiology \& Performance 1(1): 50-57.

Borg, G. (1998). Borg's perceived exertion and pain scales. Champaign, IL., Human Kinetics.

Cohen, J. (1988). Statistical power analysis for the behavioural sciences. Hillsdale, NJ, Lawrence Erlbaum.

Curran-Everett, D. and D. J. Benos (2004). "Guidelines for reporting statistics in journals published by the American Physiological Society." Physiological Genomics 18(3): 249-251.

Craig, H. (1957). Isotopic standards for carbon and oxygen and correction factors. Geochimica et Cosmochimica Acta 12: 133-149.

Ferrannini, E. (1988). The theoretical bases of indirect calorimetry: a review. Metabolism 37(3): 287-301.

Frankovich, R. J. and C. M. Lebrun (2000). Menstrual cycle, contraception, and performance. Clinics in Sports Medicine 19(2): 251-271.

Goedecke, J., V. R. Clark, et al. (2005). The effects of medium-chain triacylglycerol and carbohydrate ingestion on ultra-endurance exercise performance. International Journal of Sport Nutrition \& Exercise Metabolism 14: 15-27.

Goedecke, J. H., R. Elmer-English, et al. (1999). Effects of medium-chain triacylglycerol ingested with carbohydrate on metabolism and exercise performance. International Journal of Sport Nutrition 9: 35-47. 
Greenberger, N., J. Rodgers, et al. (1966). Absorption of medium and long chain triglycerides: Factors influencing their hydrolysis and transport. Journal of Clinical Investigation 45: 217-227.

Hopkins, W. G. (2001, Dec 2001). Calculating likely (confidence) limits and likelihoods for true values (Excel spreadsheet). In: A new view of statistics. 2007, from www.sportsci.org/resource/stats/xcl.xls.

Jeukendrup, A., J. Thielen, et al. (1998). Effect of medium-chain triacylglycerol and carbohydrate ingestion during exercise on substrate utilization and subsequent cycling performance. American Journal of Clinical Nutrition 67: 397-404.

Jeukendrup, A. and G. Wallis (2005). Measurement of substrate oxidation during exercise by means of gas exchange measurements. International Journal of Sports Medicine 26(Suppl. 1): S1-S10.

Jeukendrup, A. E., W. H. Saris, et al. (1995). Metabolic availability of medium-chain triglycerides coingested with carbohydrates during prolonged exercise. Journal of Applied Physiology 79(3): 756-62.

Jeukendrup, A. E., W. H. Saris, et al. (1996). Effect of endogenous carbohydrate availability on oral medium-chain triglyceride oxidation during prolonged exercise. Journal of Applied Physiology 80(3): 949-54.

Jeukendrup, A. E., W. H. M. Saris, et al. (1995). Metabolic availability of medium-chain triglycerides coingested with carbohydrates during prolonged exercise. Journal of Applied Physiology 79: 756-762.

Kanaley, J. A., R. A. Boileau, et al. (1992). Substrate oxidation and GH responses to exercise are independent of menstrual phase and status. Medicine \& Science in Sports \& Exercise 24(8): 873-80. 
Kiens, B. and J. W. Helge (1998). Effect of high-fat diets on exercise performance. Proceedings of the Nutrition Society 57: 73-75.

Livesey, G. and M. Elia (1988). Estimation of energy expenditure, net carbohydrate utilization, and net fat oxidation and synthesis by indirect calorimetry: evaluation of errors with special reference to the detailed composition of fuels. 608-628. American Journal of Clinical Nutrition 47: 608-628.

Misell, L., N. Lagomarcino, et al. (2001). Chronic medium-chain triacylglycerol consumption and endurance performance in trained runners. Journal of Sports Medicine and Physical Fitness 41: 210-215.

Misell, L. M., N. D. Lagomarcino, et al. (2001). Chronic medium-chain triacylglycerol consumption and endurance performance in trained runners. Journal of Sports Medicine and Physical Fitness 41(2): 210-215.

Mott, C., H. Sarles, et al. (1972). Different action of short, medium, and long chain triglycerides on exocrine pancreatic secretion in man. Biologie et GastroEnterologie 5(1): 79-84.

Oopik, V., S. Timpmann, et al. (2001). Effects of daily medium-chain triglyceride ingestion on energy metabolism and endurance performance capacity in well-trained runners. Nutrition Research 21(8): 1125-1135.

Paton, C. D. and W. G. Hopkins (2005). Variation in performance of elite cyclists from race to race. European Journal of Sport Science: 1-7.

Patterson, B. W., G. Zhao, et al. (1999). Validation of a new procedure to determine plasma fatty acid concentration and isotopic enrichment. Journal of Lipid Research 40: 2118-2124. 
Péronnet, F. and D. Massicotte (1991). Table of non-protein respiratory quotient: An update. Canadian Journal of Sport Sciences 161(1): 23-29.

Robert, J. J., Koziet, J., Chauvet, D., Darmaun, D., Desjeux, J.F. and Young, V.R. (1987). Use of $13 \mathrm{C}$ labelled glucose for estimating glucose oxidation: some design considerations. Journal of Applied Physiology 63: 1725-1732.

Rowlands, D. and W. Hopkins (2002). Effect of high-fat, high-carbohydrate, and highprotein meals on metabolism and performance during endurance cycling. International Journal of Sport Nutrition and Exercise Metabolism. 12: 318-335.

Sidossis, L., A. Gastaldelli, et al. (1997). Regulation of plasma fatty acid oxidation during low- and high-intensity exercise. American Journal of Physiology: Endocrinology and Metabolism. 272(6): E1065-E1070.

Sterne, J. A. C. and G. D. Smith (2001). "Sifting the evidence-what's wrong with significance tests?" British Medical Journal 322: 226-261.

Tarnopolsky, M. A. (2000). Gender differences in metabolism; nutrition and supplements. [Review] [49 refs]. Journal of Science \& Medicine in Sport 3(3): 287-98.

Tarnopolsky, M. A. (2000). Gender differences in substrate metabolism during endurance exercise. Canadian Journal of Applied Physiology 25(4): 312-327.

Thorburn, M. S., B. Vistisen, et al. (2006). Attenuated gastric tolerance but no benefit to performance with adaptation to octanoate-rich esterified oils in well-trained male cyclists. Journal of Applied Physiology 101: 1733-1743.

Van Hall, G., J. Bulow, et al. (2002). Regional fat metabolism in human splanchnic and adipose tissues: the effect of exercise. Journal of Physiology 543: 1033-1046. 
Van Zyl, C. G., E. V. Lambert, et al. (1996). Effects of medium-chain triglyceride ingestion on fuel metabolism and cycling performance. Journal of Applied Physiology 80(6): 2217-25.

VanZyl, C., E. Lambert, et al. (1996). Effects of medium-chain triglyceride ingestion on fuel metabolism and cycling performance. Journal of Applied Physiology 80(6): $2217-2225$.

Vistisen, B., L. Nybo, et al. (2003). Minor amounts of plasma medium-chain fatty acids and no improved time trial performance after consuming lipids. Journal of Applied Physiology 95(6): 2434-2443.

Vukovich, M., D. Costill, et al. (1993). Effect of fat emulsion, infusion and fat feeding on muscle glycogen utilisation during exercise. Journal of Applied Physiology 75: 1513-1518. 


\section{Tables}

Table 1. Comparison of the three dietary conditions on performance.

\begin{tabular}{lccc}
\hline & \multicolumn{2}{c}{ Effect Comparisons $(\%) \pm 90 \% \mathrm{CL}^{\mathrm{a}}$ and Qualitative Inference } \\
PERFORMANCE & MC-MC minus & LC-MC minus & MC-MC minus \\
MEASURE & LC-CHO & LC-CHO & LC-MC
\end{tabular}

Sprints 1-9

$\begin{array}{lccc}\text { Mean Power }^{\mathrm{b}} & -1.1 \pm 3.9 & -1.9 \pm 8.5 & 0.9 \pm 9.5 \\ & \text { Unclear } & \text { Unclear } & \text { Unclear } \\ \text { Fatigue }^{\mathrm{c}} & -0.9 \pm 6.4 & -0.4 \pm 5.3 & 0.4 \pm 6.1 \\ & \text { Unclear } & \text { Unclear } & \text { Unclear }\end{array}$

Sprint 10

$\begin{array}{llll}\text { Mean Power } & -2.2 \pm 6.7 & -2.8 \pm 9.2 & 0.6 \pm 9.6\end{array}$

Unclear Unclear Unclear

${ }^{a} \pm 90 \%$ CL: add and subtract this number to the mean effect to obtain the $90 \%$ confidence limits for the true difference.

${ }^{b}$ Mean power is the overall mean power effect for sprints 1-9. The sprint 10 effect was excluded in the linear model because is approached differently by the subjects; it was extracted separately from the repeated-measures model.

${ }^{c}$ Fatigue is the percentage difference between the conditions in the linear decline in 
power evaluated between sprints 1 and 9. Analysis is based on the reduction in mean power per sprint. More negative means greater relative fatigue.

Abbreviations: MCFA-rich esterified oil diet with the CHO+MCFA-rich oil emulsion ingested during exercise (MC-MC); long-chain fatty-acid rich diet with the $\mathrm{CHO}+\mathrm{MCFA}$-rich esterified oil emulsion ingested during exercise (LC-MC); long-chain fatty-acid rich diet with a CHO-only during exercise (LC-CHO). 
Table 2. Summary of the overall effect of the three dietary conditions on gastrointestinal distress and exertional parameters during the 3-h ride and performance test.

\begin{tabular}{|c|c|c|c|c|c|c|}
\hline \multirow{5}{*}{ PARAMETER } & \multicolumn{6}{|c|}{ Mean Effect Comparisons ${ }^{\mathrm{a}} \pm 90 \% \mathrm{CL}^{\mathrm{b}}$ and Qualitative Inference } \\
\hline & \multicolumn{3}{|c|}{ 3-h Ride } & \multicolumn{3}{|c|}{ Performance Test } \\
\hline & $\mathrm{MC}-\mathrm{MC}$ & LC-MC minus & $\mathrm{MC}-\mathrm{MC}$ & MC-MC minus & LC-MC minus & MC-MC \\
\hline & minus & $\mathrm{LC}-\mathrm{CHO}$ & minus & $\mathrm{LC}-\mathrm{CHO}$ & $\mathrm{LC}-\mathrm{CHO}$ & minus \\
\hline & $\mathrm{LC}-\mathrm{CHO}$ & & LC-MC & & & LC-MC \\
\hline \multirow[t]{3}{*}{ Nausea } & $0.8 \pm 0.5$ & $0.4 \pm 0.5$ & $0.4 \pm 0.8$ & $2.5 \pm 0.7$ & $2.1 \pm 0.5$ & $0.3 \pm 0.6$ \\
\hline & Increase very & Increase & Unclear & Increase almost & Increase almost & Increase \\
\hline & likely & possible & & certain & certain & unlikely \\
\hline Fullness / & $1.9 \pm 2.6$ & $1.6 \pm 0.2$ & $0.4 \pm 3.1$ & $3.5 \pm 1.9$ & $2.7 \pm 0.4$ & $0.8 \pm 2.7$ \\
\hline Bloating & Unclear & Increase & Unclear & Increase very likely & Increase almost & Unclear \\
\hline
\end{tabular}




\begin{tabular}{|c|c|c|c|c|c|c|}
\hline & & almost certain & & & certain & \\
\hline Stomach & $0.6 \pm 1.4$ & $0.3 \pm 0.2$ & $0.4 \pm 1.6$ & $0.6 \pm 1.8$ & $0.9 \pm 1.5$ & $-0.2 \pm 2.4$ \\
\hline Cramp & Unclear & Increase likely & Unclear & Unclear & Unclear & Unclear \\
\hline \multirow[t]{3}{*}{ Reflux } & $1.8 \pm 1.3$ & $1.7 \pm 0.2$ & $0.0 \pm 1.6$ & $2.6 \pm 0.4$ & $3.3 \pm 0.5$ & $-0.6 \pm 0.9$ \\
\hline & Increase very & Increase & Unclear & Increase almost & Increase almost & Decrease \\
\hline & likely & almost certain & & certain & certain & possible \\
\hline \multirow[t]{3}{*}{ Leg Soreness } & $0.1 \pm 0.4$ & $0.2 \pm 0.3$ & $-0.1 \pm 0.3$ & $0.4 \pm 1.7$ & $1.9 \pm 0.5$ & $-1.5 \pm 1.6$ \\
\hline & Unclear & Increase & Decrease & Unclear & Increase almost & Decrease \\
\hline & & possible & unlikely & & certain & likely \\
\hline Perceived & $0.3 \pm 0.2$ & $0.1 \pm 0.2$ & $0.2 \pm 0.2$ & $0.8 \pm 1.3$ & $1.3 \pm 0.6$ & $-0.5 \pm 1.3$ \\
\hline \multirow[t]{2}{*}{ Effort } & Increase likely & Increase & Increase & Increase possible & Increase very likely & Unclear \\
\hline & & possible & possible & & & \\
\hline
\end{tabular}

${ }^{\mathrm{a}}$ Mean effect comparisons are presented in Likert Scale Units

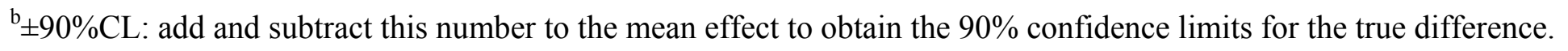

Abbreviations: MCFA-rich esterified oil diet with the $\mathrm{CHO}+\mathrm{MCFA}-$ rich oil emulsion ingested during exercise (MC-MC); long-chain 
fatty-acid rich diet with the $\mathrm{CHO}+\mathrm{MCFA}$-rich esterified oil emulsion ingested during exercise (LC-MC); long-chain fatty-acid rich diet with a CHO-only during exercise (LC-CHO). 


\section{Figure Captions}

Figure 1. Study Design. Three 2-week dietary adaptation periods including a ${ }^{13} \mathrm{C}$ background breath-enrichment trial on day 11 and performance test on day 14 with ingestion of either the CHO+MCFA-rich esterified oil emulsion or placebo CHO-only exercise supplement.

Figure 2. Sprint mean power in the performance tests. Data are back log-transformed least squares means. Bars are between-subject $\mathrm{SD}_{\mathrm{f}}$, those marked with an $\times$ represent the withinsubject deviation. Y-axes are presented on logarithmic scales.

Figure 3. Effect of covariate nausea on mean sprint power (y-axis position coefficient) derived from the within-subject polynomial analysis. Data points are the observed mean effect of treatment relative to the reference condition and bars are $90 \%$ confidence intervals. The proportion of the confidence interval with beneficial, trivial or detrimental population outcomes is defined and the practical inference of the observed outcome qualified on the right.

Figure 4. Breath ${ }^{13} \mathrm{C}$-enrichment. Data are raw means \pm between-subject SD.

Figure 5. Mean substrate oxidation as percentage contribution to total energy expenditure during the 3 -h rides. 
Figure 6. Pattern of substrate oxidation during the 3-h rides. Data are back-log transformed least-squares means. Bars are between-subject $\mathrm{SD}_{\mathrm{f}}$, those marked with an $\times$ represent the within-subject deviation. Y-axes are presented on logarithmic scales.

Figure 7. Exertional parameters and gastrointestinal distress ratings during the 3 -h and performance tests. Ratings are least-squares means \pm between-subject SD. SD bars marked with an $\times$ represent the within-subject deviation.

Figure 8. Effect of treatment on plasma lipid concentration during exercise. Octanoic acid closed symbols $=$ total octanoic acid; open symbols $={ }^{13} \mathrm{C}$-enriched octanoic acid. Bars are between-subject $\mathrm{SD}_{\mathrm{f} .} \mathrm{SD}$ bars marked with an $\times$ represent the within-subject deviation. Yaxes are presented on logarithmic scales. 


\section{Illustrations}

Figure 1.

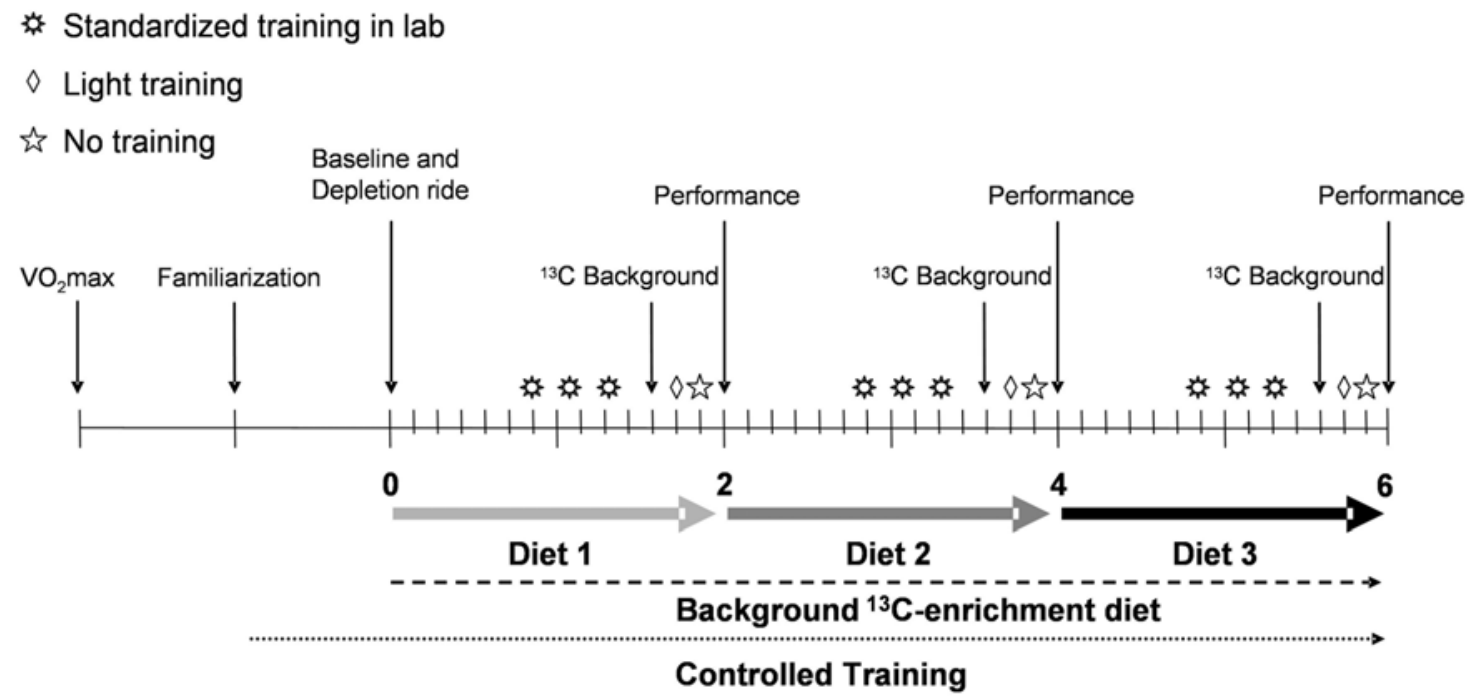


Figure 2.

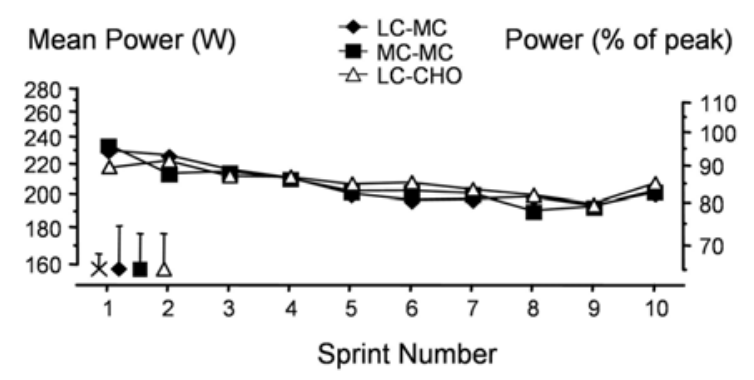


Figure 3.

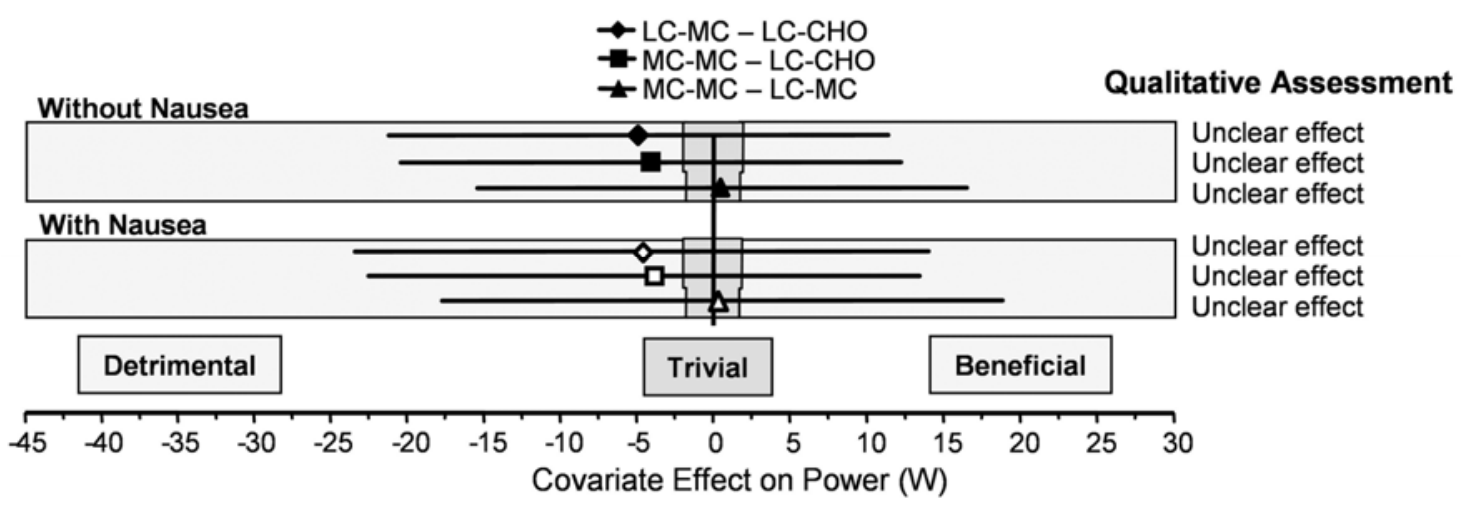


Figure 4 .

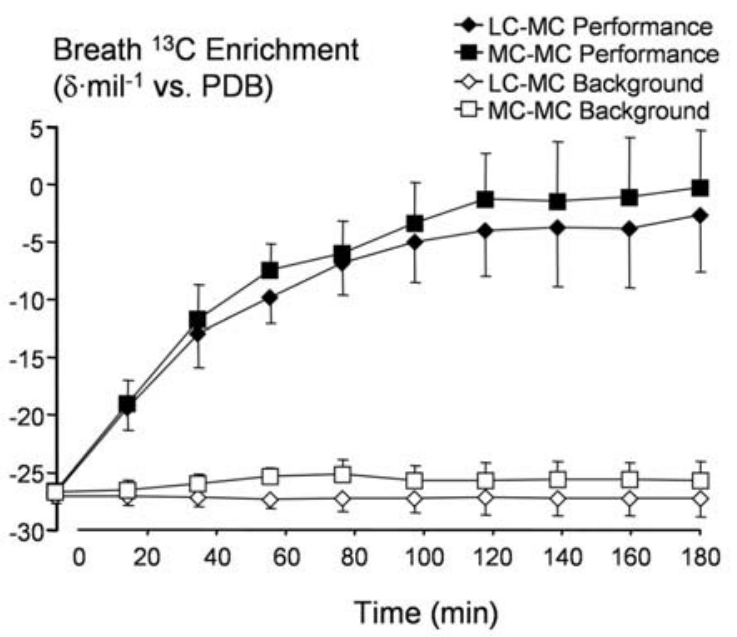


Figure 5.

Contribution to total

energy expenditure (\%)

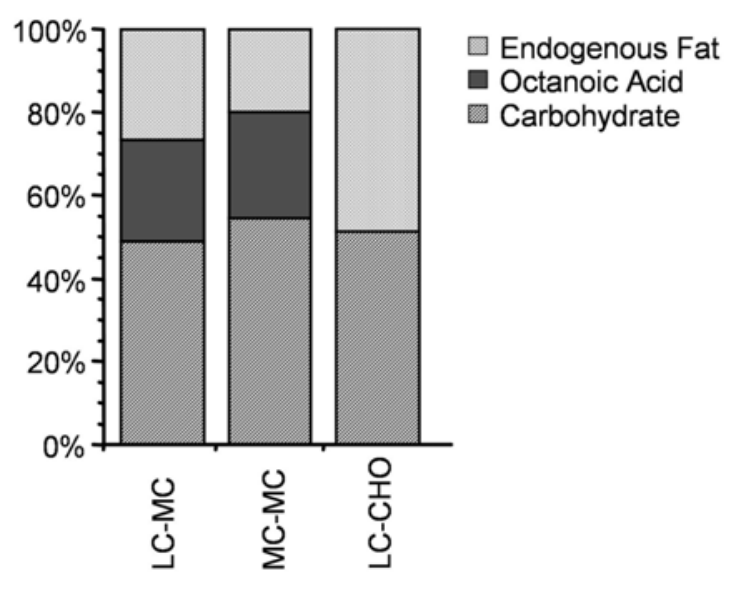


Figure 6 .
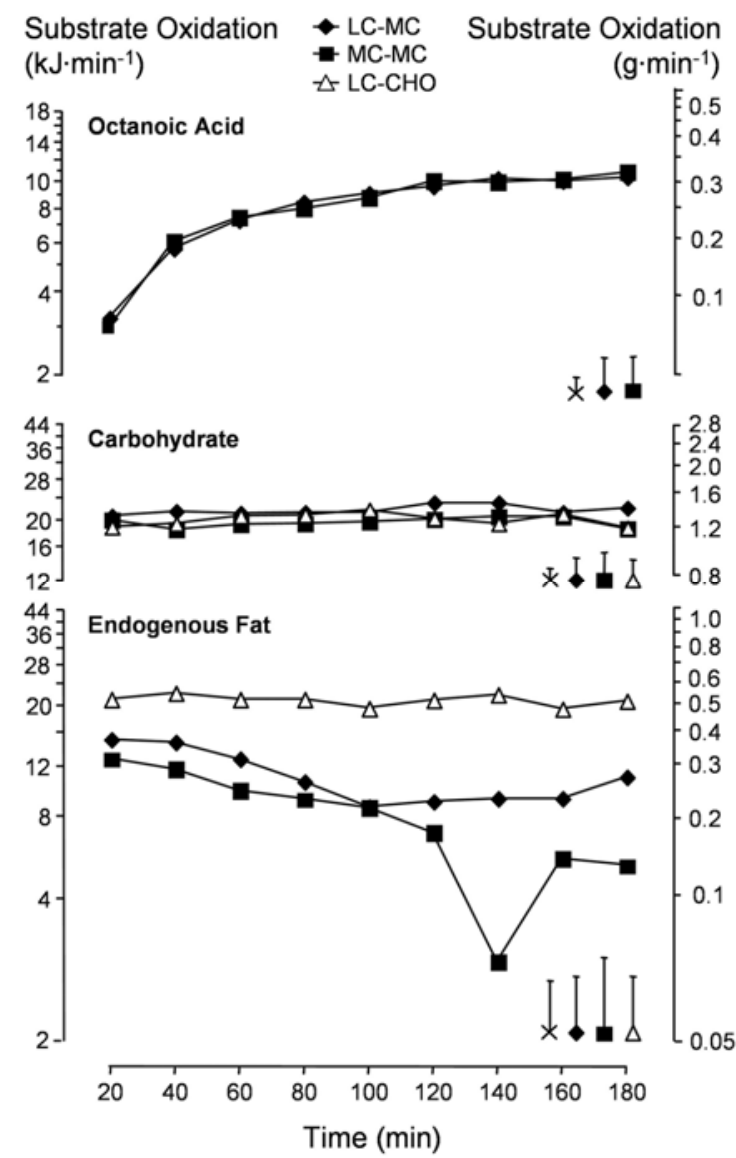
Figure 7.

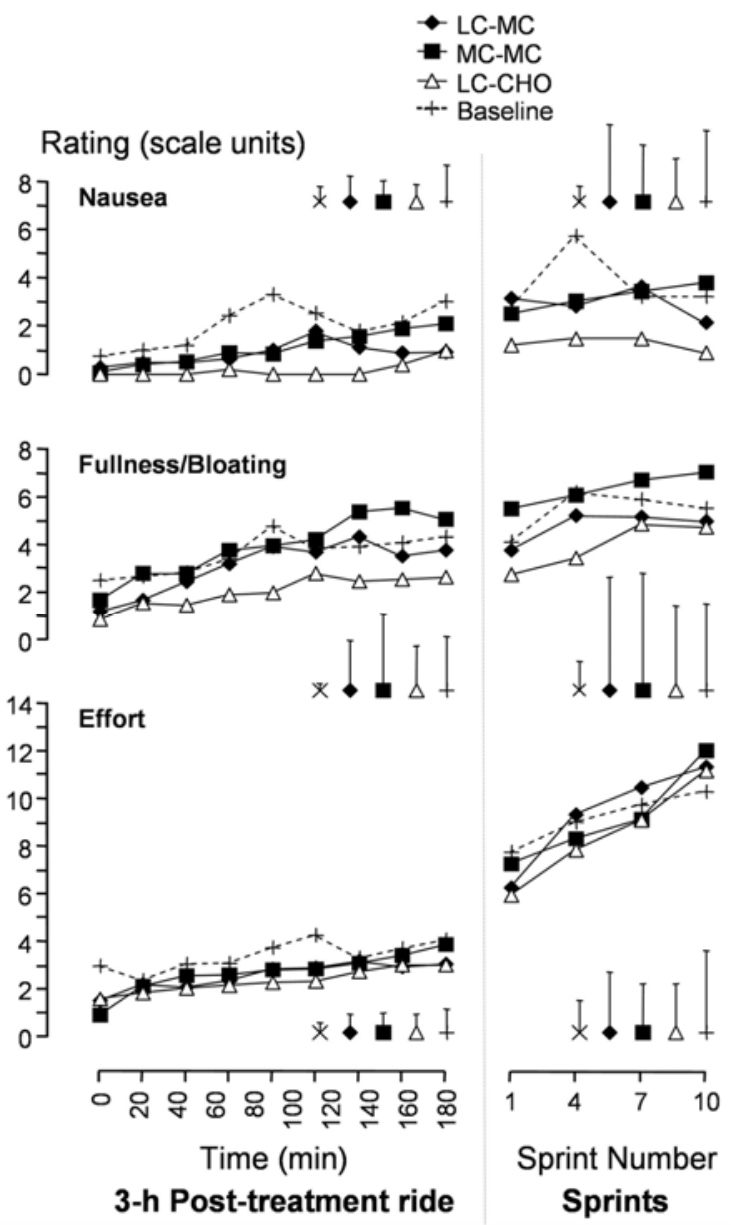


Figure 8.

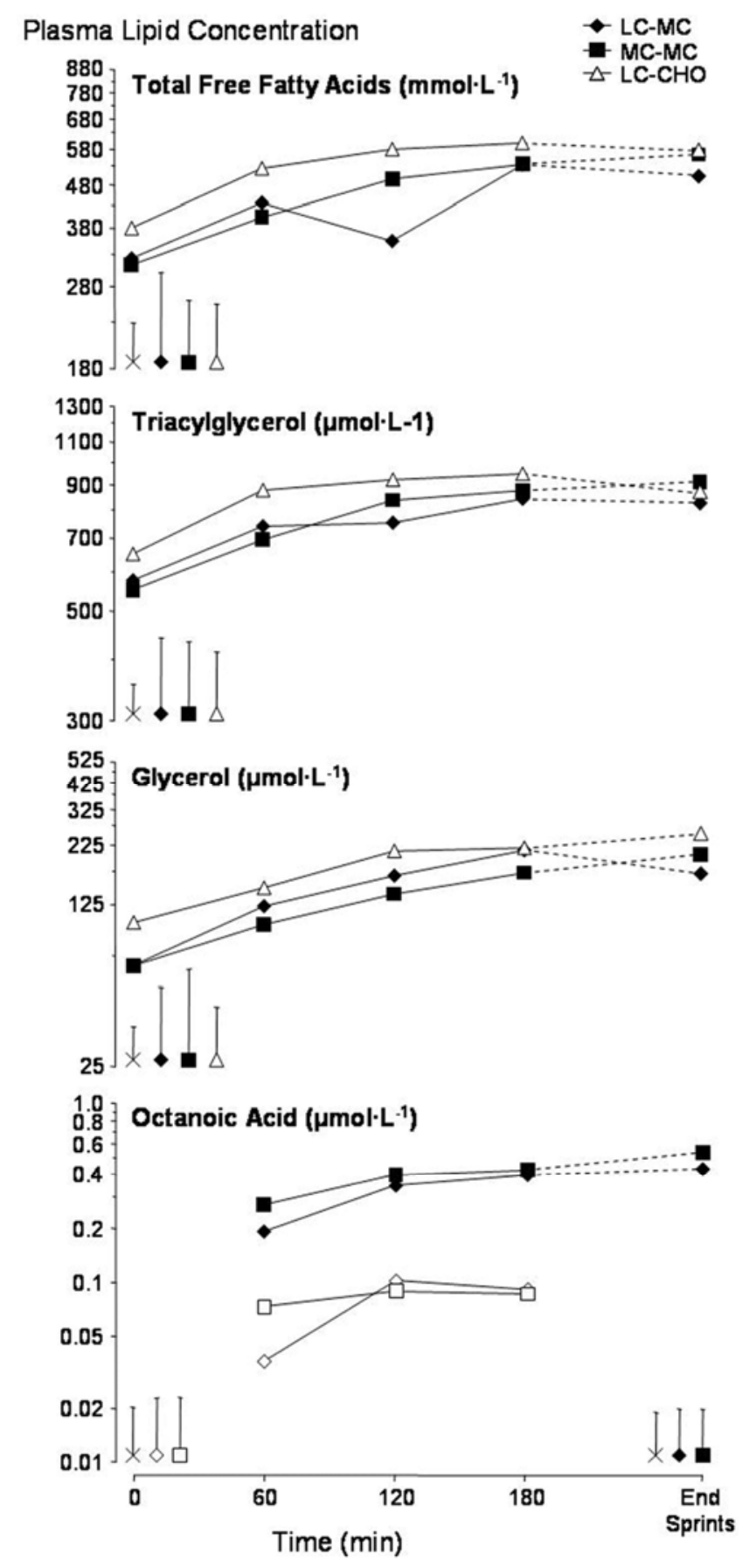

